\title{
The influence of microlensing on spectral line shapes generated by a relativistic accretion disc
}

\author{
L. Č. Popović ${ }^{1}$, E. G. Mediavilla ${ }^{2}$, and J. A. Muñoz ${ }^{2}$ \\ 1 Astronomical Observatory, Volgina 7, 11160 Belgrade 74, Serbia, Yugoslavia \\ 2 Instituto de Astrofísica de Canarias, 382005 La Laguna, Tenerife, Spain
}

Received 2 March 2001 / Accepted 8 August 2001

\begin{abstract}
We study the influence of gravitational microlensing on the spectral line profiles originating from a relativistic accretion disc. Using the Chen \& Halpern model for the disc, we show the noticeable changes that microlensing can induce in the line shape when the Einstein radius associated with the microlens is of a size comparable to that of the accretion disc. Of special interest is the relative enhancement between the blue and red peaks of the line when an off-center microlens affects the approaching and receding parts of the accretion disc asymmetrically.

In an AGN formed by a super-massive binary in which the accretion disc is located around one of the supermassive companions (the primary), we discuss the possibility of microlensing by the secondary. In this case the ratio between the blue and red peaks of the line profile would depend on the orbital phase. We have also considered the more standard configuration of microlensing by a star-sized object in an intervening galaxy and find that microlensing may also be detected in the broad emission lines of multiply imaged QSOs. The changes observed in the line profile of Arp 102 B are taken as a reference for exploring both scenarios.
\end{abstract}

Key words. accretion - accretion disks - line: formation - relativity - gravitational lensing

\section{Introduction}

The influence of microlensing effects on multiply imaged QSOs has been widely discussed (Ostriker \& Vietri 1985; Kayser et al. 1986; Schneider \& Weiss 1987; Nemiroff 1988; Irwin et al. 1989; Schneider \& Wambsganss 1990; Yonehara et al. 1999; Mineshige \& Yonehara 1999; Agol \& Krolik 1999; Kraus et al. 1999; Belle \& Lewis 2000; Abajas et al. 2001). It has been routinely assumed that only the continuum-emitting region of Active Galactic Nuclei (AGNs) is sufficiently compact to be affected by microlensing. However, recent results (e.g. Wandel et al. 1999) indicate that broad line regions (BLRs) of AGNs are smaller than was supposed. According to these new measurements, gravitational microlensing by stars in the lens galaxy can affect not only the continuum but also the broad emission lines of multiply imaged AGN (Abajas et al. 2001).

One proposed model for BLRs is that of a compact accretion disc, whose signature would be the existence of

Send offprint requests to: L. Č. Popović, e-mail: lpopovic@aob.bg.ac.yu double-peaked broad emission line profiles (e.g. Chen \& Halpern 1989; Chen et al. 1989; Eracleous \& Halpern 1994; Newman et al. 1997; Eracleous et al. 1997; Corbett et al. 1998; Eracleous 1999, Popović et al. 2001). This signature is found in a number of AGNs, mainly radio-loud objects (in particular, about 10-15\% of AGN with redshift $z<0.4$ have double-peaked lines; Eracleous 1999). Typical examples are 3C 390.3 (Perez et al. 1988; Dietrich et al. 1998) and Arp 102 B (Chen \& Halpern 1989; Halpern et al. 1996, Sergeev et al. 2000). The aim of this article is to study the influence of microlensing on the double-peaked emission lines generated by an accretion disc.

However, the disc model by itself cannot explain the independent variation of the blue and red peaks of the lines found over long time-scales (Sulentic 1999), as e.g. the variation in $\mathrm{H}_{\alpha}$ line of Arp102B was about $16 \%$ in the 1990-1995 period (Newman et al. 1997). Additional effects (Zheng et al. 1991; Newman et al. 1997) or alternative models (Sergeev et al. 2000) have been proposed to explain this behavior of the double-peaked spectral lines. In this article we will show that, with an adequate scenario, the selective amplification induced by microlensing can also give rise to this effect. 
In Sect. 2 we include the microlensing by a compact object in the relativistic disc model of Chen \& Halpern (1989) and analyse the consequences in Sect. 3. In Sect. 4 two different physical stages are explored as alternatives to explain the changes observed in the line profile of Arp 102 B in terms of microlensing.

\section{Relativistic accretion disc model incorporating microlensing}

We include microlensing in the relativistic disc model developed by Chen et al. (1989) and Chen \& Halpern (1989). For the model parameters we take as reference values those proposed by the same authors for the typical radioloud AGN, Arp 102 B. The double-peaked line profiles of this object are very well fitted by the relativistic accretion disc model in the periods before 1990 and after 1995 (Chen \& Halpern 1989; Newman et al. 1997). Thus, we will adopt a disc with inclination $i=32^{\circ}$, inner radius $\xi_{\text {in }}=350 \Re_{\mathrm{g}}$, outer radius $\xi_{\text {out }}=1000 \Re_{\mathrm{g}}$, where $\Re_{\mathrm{g}}=G M / c^{2}=1.4745 \times 10^{11} M_{8}$ is the gravitational radius, and $M_{8}$ is the mass in units of $10^{8} M_{\odot}$. The emissivity as a function of radius is given by

$\epsilon=\epsilon_{0} \xi^{-p}$,

with $p=3$ for the case of Arp 102 B (Chen \& Halpern 1989).

Including the gravitational microlensing effects in the relativistic accretion disc model the line shape will be

$$
\begin{aligned}
\frac{F_{x}}{F_{0}}= & \int_{\xi_{\text {in }}}^{\xi_{\text {out }}} \int_{-\pi / 2}^{\pi / 2} \mathrm{e}^{-\frac{(1+x-D(\xi, \phi))^{2} \lambda_{0}^{2}}{2 \sigma^{2} D^{2}(\xi, \phi)}} D^{3}(\xi, \phi) \\
& \times \xi^{1-p} g(\xi, \phi) \cdot A(\xi, \phi) \mathrm{d} \xi \mathrm{d} \phi
\end{aligned}
$$

where $\lambda_{0}$ is the transition wavelength; $\sigma$ is the velocity dispersion and $x=\lambda_{0} / \lambda$. In the case of Arp $102 \mathrm{~B} \sigma / \lambda_{0}$ corresponds to a velocity of $850 \mathrm{~km} \mathrm{~s}^{-1}$ (Chen \& Halpern 1989).

The $F_{0}$ in Eq. (1) is (Chen \& Halpern 1989)

$F_{0}=\frac{2 \epsilon_{0} M^{2} \cos i}{4 \pi \sqrt{2 \pi} d^{2}} \frac{\lambda_{0}}{\sigma}$

where $M$ is the mass of black hole, $d$ is the distance to the AGN.

The functions $D$ and $g$ in Eq. (1) are,

$$
D(\xi, \phi)=\sqrt{(1-3 / \xi)}\left(1+\frac{\sin i \sin \phi}{\sqrt{\xi}}\right)^{-1}
$$

where

$$
\begin{aligned}
g(\xi, \phi)= & 1-\frac{1}{\xi} \\
& +\frac{1}{\xi} \frac{2 D^{2}(\xi, \phi)}{D^{2}(\xi, \phi) \cos ^{2} i+\xi[D(\xi, \phi)-\sqrt{1-3 / \xi}]^{2}} .
\end{aligned}
$$

The amplification caused by microlensing is given by the function

$A(\xi, \phi)=\frac{u^{2}(\xi, \phi)+2}{u(\xi, \phi) \sqrt{u^{2}(\xi, \phi)+4}}$, where $u(\xi, \phi)$ corresponds to the angular separation between lens and source in the unit of the Einstein ring radius and it is obtained from

$u(\xi, \phi)=\frac{\sqrt{\left(\xi \cos \phi \cos i-\xi_{0} \cos \phi_{0}\right)^{2}+\left(\xi \sin \phi-\xi_{0} \sin \phi_{0}\right)^{2}}}{\eta_{0}}$,

$\xi_{0}$ being the radial distance of the microlens from the disc center, $\phi_{0}$ the azimuthal position of the microlens and $\eta_{0}$ the Einstein ring radius expressed in gravitational radii, $\Re_{\mathrm{g}}$, units.

\section{Results}

In first place, we have computed the line profile when a microlens of Einstein radius $\eta_{0}=500 \Re_{\mathrm{g}}$ is projected on to the center of the accretion disc, $\xi_{0}=0$. As can be seen in Figure 1 the intensity of the spectral line is amplified, but the line shape is not deformed. However, off-centered microlenses would asymmetrically amplify the red and blue parts of the line profile.

To investigate the influence of the location of the microlens with respect to the accretion disc, we consider three different cases.

\subsection{Radial displacement along the minor axis, $\phi_{0}=0$}

If we locate the microlens in the minor axis $\left(\phi_{0}=0\right)$ at a radial distance $\xi_{0}=500 \Re_{\mathrm{g}}$ we notice a selective amplification of the line profile in the region around zero velocities (see Fig. 2). This amplification increases for increasing values of the Einstein radius of the microlens and can be observed for values above $\eta_{0}=40 \Re_{\mathrm{g}}$ (for the considered disk). For higher values it can give rise to a central peak in the line profile (Fig. 2).

In Fig. 3 we present the ratio of red- to blue-peak intensities $\left(R=I_{\text {red }} / I_{\text {blue }}\right)$ as a function of the radial position of the microlens along the minor axis. According to this figure, radial displacements along the minor axis would result in only small changes of this quotient.

\subsection{Azimuthal displacement at constant radius}

In this case we compute the line profile for different values of the azimuthal position of the microlens, $\phi_{0}=$ $-90^{\circ},-45^{\circ}, 0^{\circ}, 45^{\circ}$ and $90^{\circ}$. We present the resulting line profiles in Figs. 4 to 8 for a microlens of projected Einstein radius $\eta_{0}=500 \Re_{\mathrm{g}}$ and located at a distance $\xi_{0}=500 \Re_{\mathrm{g}}$. As can be seen, the changes in $\phi_{0}$ induce asymmetrical enhancements in the red/blue peaks. To quantify the relevance of this effect, in Table 1 we present the ratio of red- to blue-peak intensities $(R)$ for different values of microlens masses (i.e. for different Einstein ring radii) and azimuthal positions. For an unperturbed profile the ratio is $R=0.834$. According to Table $1, R$ undergoes significant changes in function of the azimuthal position as well as of the Einstein radius of the microlens. 


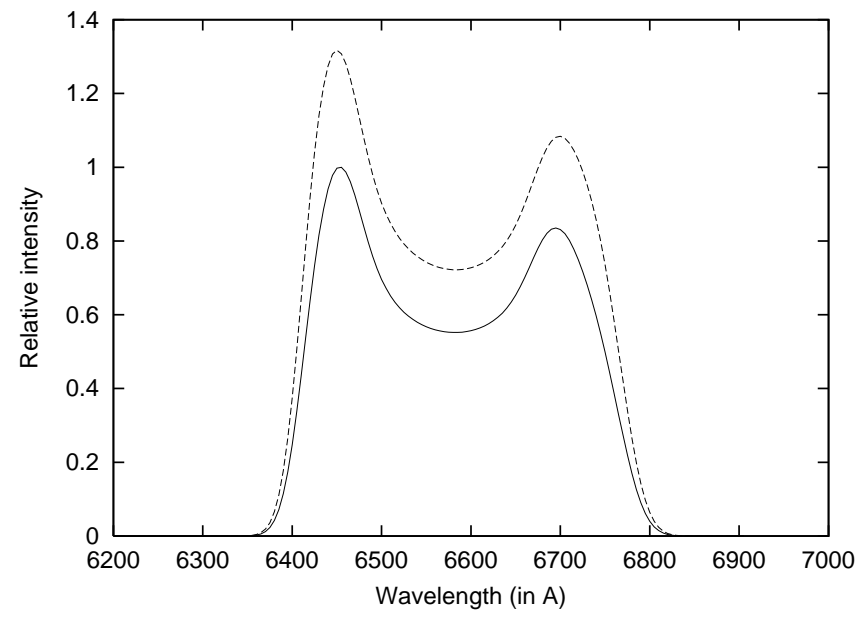

Fig. 1. The $\mathrm{H}_{\alpha}$ line profile calculated for a relativistic disc with parameters $i=32^{\circ}, \xi_{\text {in }}=350 \Re_{\mathrm{g}}, \xi_{\text {out }}=1000 \Re_{\mathrm{g}}$ and $\sigma / \lambda_{0}=850 \mathrm{~km} \mathrm{~s}^{-1}$. The profile deformed by gravitational microlensing is represented by the dashed line. The parameters of the gravitational microlens are: $\xi_{0}=0 \Re_{\mathrm{g}}, \eta_{0}=500 R_{\mathrm{g}}$ and $\phi_{0}=90^{\circ}$. The unperturbed profile is represented by the solid line.

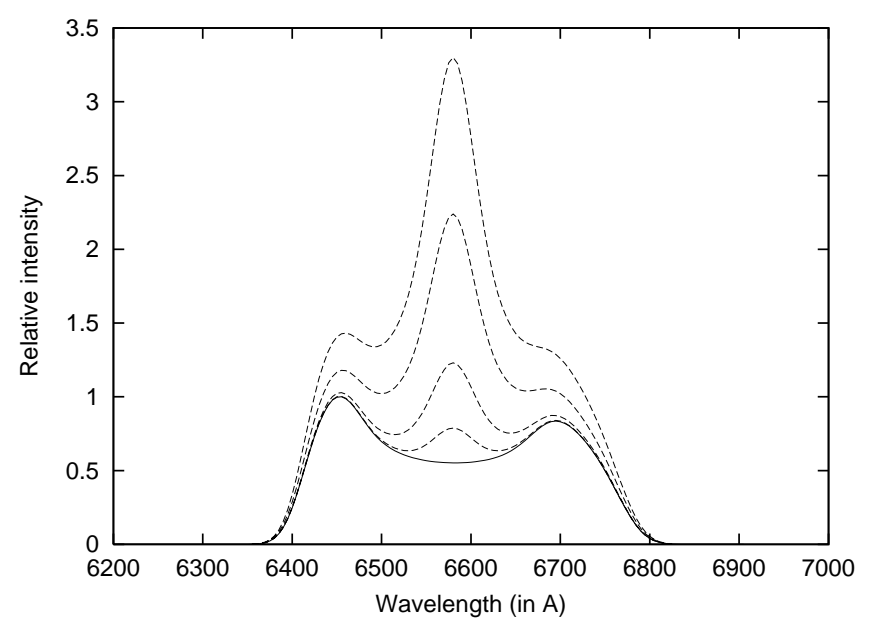

Fig. 2. The line profiles for different Einstein ring radii of the lenses $\eta_{0}=100 \Re_{\mathrm{g}}, 200 \Re_{\mathrm{g}}, 400 \Re_{\mathrm{g}}$ and $600 \Re_{\mathrm{g}}$, respectively. The intensity of the central part is higher for higher $\eta_{0}$. The calculations were performed for $\xi_{0}=500 \Re_{\mathrm{g}}$ and $\phi_{0}=0^{\circ}$.

In Figs. 9 and 10 we represent the changes in the parameter $R$ as a function of the azimuthal position of the microlens for $\eta_{0}=150 \Re_{\mathrm{g}}$ and $300 \Re_{\mathrm{g}}$. We have also represented the best sinusoidal fit according to the function

$R\left(\phi_{0}\right)=C_{1}+C_{2} \cdot \phi_{0} \cos \phi_{0}$,

where $C_{1}$ and $C_{2}$ are constants. For $\eta_{0}=150 \Re_{\mathrm{g}}$ we obtained $C_{1}=0.9135$ and $C_{2}=0.0126$ (Fig. 9), and for $\eta_{0}=300 \Re_{\mathrm{g}}, C_{1}=1.162$ and $C_{2}=0.0316$ (Fig. 10). The amplitude of the variation decreases in the direction $\phi \rightarrow-90^{\circ}$.

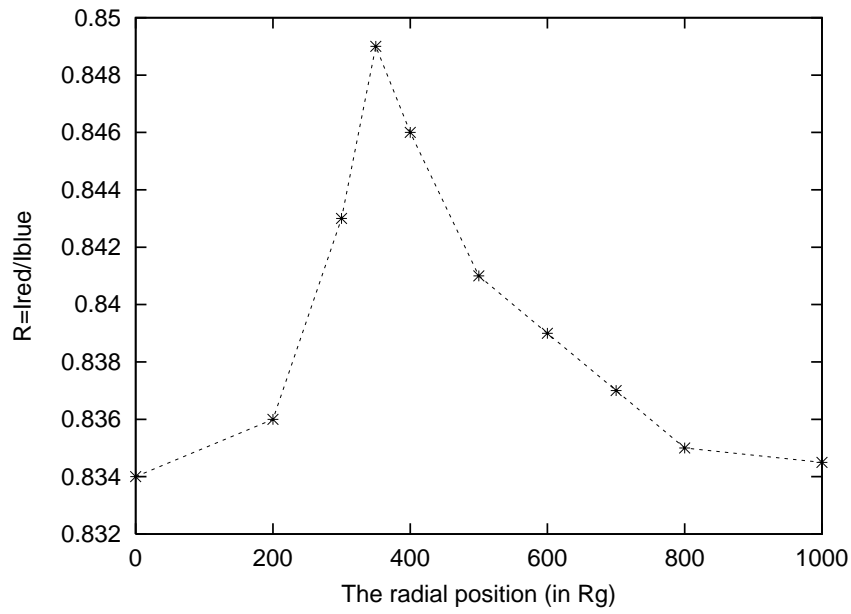

Fig. 3. The ratio of red to blue intensities $(R)$ as a function of radial position of the microlens $\left(\xi_{0}\right)$. The calculations were performed for a relativistic disc with parameters $i=32^{\circ}$, $\xi_{\text {in }}=350 \Re_{\mathrm{g}}, \xi_{\text {out }}=1000 \Re_{\mathrm{g}}$ and $\sigma / \lambda_{0}=850 \mathrm{~km} \mathrm{~s}^{-1}$. The parameters of the gravitational lens are: $\phi_{0}=0^{\circ}$ and $\eta_{0}=150 \Re_{\mathrm{g}}$.

Table 1. The ratio of red- to blue-peak intensities $R=$ $I_{\text {red }} / I_{\text {blue }}$ for different values of the Einstein ring radius and azimuthal position of the microlens. The radial position of the microlens is $\xi_{0}=500 \Re_{\mathrm{g}}$. For an unperturbed profile $R=0.834$.

\begin{tabular}{|c|c|c|c|}
\hline$\phi_{0}$ & $R_{\eta_{0}=150 \Re_{\mathrm{g}}}$ & $R_{\eta_{0}=300 \Re_{\mathrm{g}}}$ & $R_{\eta_{0}=500 \Re_{\mathrm{g}}}$ \\
\hline \hline-90 & 0.62 & 0.39 & 0.26 \\
-45 & 0.59 & 0.37 & 0.25 \\
0 & 0.84 & 0.87 & 0.93 \\
45 & 1.47 & 2.38 & 3.06 \\
90 & 1.04 & 1.67 & 2.50 \\
\hline
\end{tabular}

\subsection{Microlens transit parallel to the major axis}

In this case, the radial position is a function of the azimuth,

$\xi_{0}=\xi_{0}^{1} / \sin \left(90-\phi_{0}\right)$,

where $\xi_{0}^{1}$ is the distance between the projected microlens trajectory and the major axis of the accretion disc. In Fig. 11 we have represented the red-to-blue intensity ratio, $R$, as a function of the azimuthal position for $\eta_{0}=200 \Re_{\mathrm{g}}$ and $\xi_{0}^{1}=350 \Re_{\mathrm{g}}$. The best sinusoidal fit, corresponding to $C_{1}=0.963$ and $C_{2}=0.0149$, is also included in Fig. 11 .

\section{Physical scenarios: the case of Arp 102 B}

Arp $102 \mathrm{~B}$ is a broad line radio galaxy at a redshift of 0.02437. The hydrogen Balmer lines of its spectrum are double-peaked and show variability in intensity and shape. For this reason, the spectra of Arp 102 has been investigated by several authors (e.g. Halpern \& Filippenko 1988; Chen \& Halpern 1989; Chen et al. 1989; Miller \& Peterson 1990; Antonucci et al. 1996; Gaskell 1996; Newman et al. 1997; Sergeev et al. 2000). As mentioned in the previous section, the shape of the emission lines of this object 


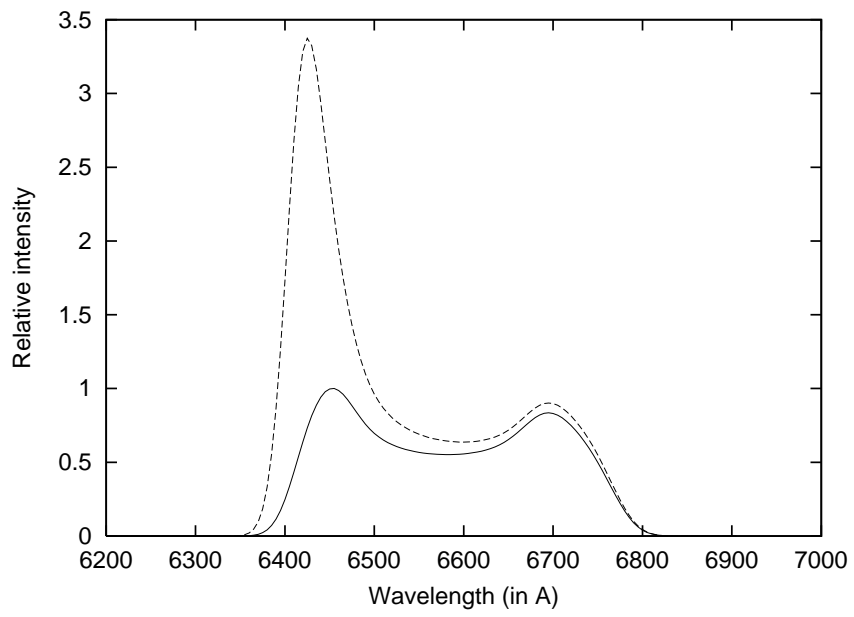

Fig. 4. The same as in Fig. 1, but the parameters of gravitational the microlens are: $\xi_{0}=500 \Re_{\mathrm{g}}, \eta_{0}=500 \Re_{\mathrm{g}}$ and $\phi_{0}=-90^{\circ}$.

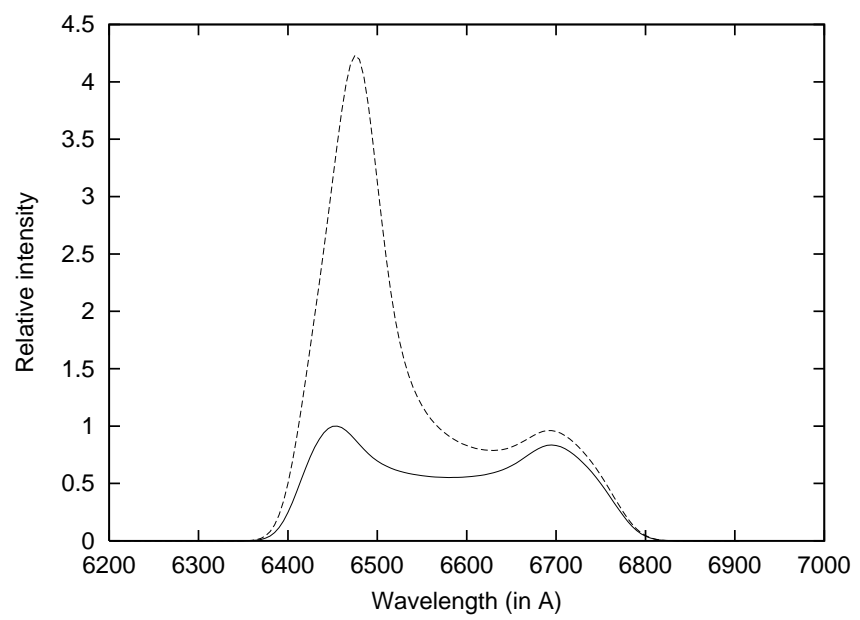

Fig. 5. The same as in Fig. 4, but for $\phi_{0}=-45^{\circ}$.

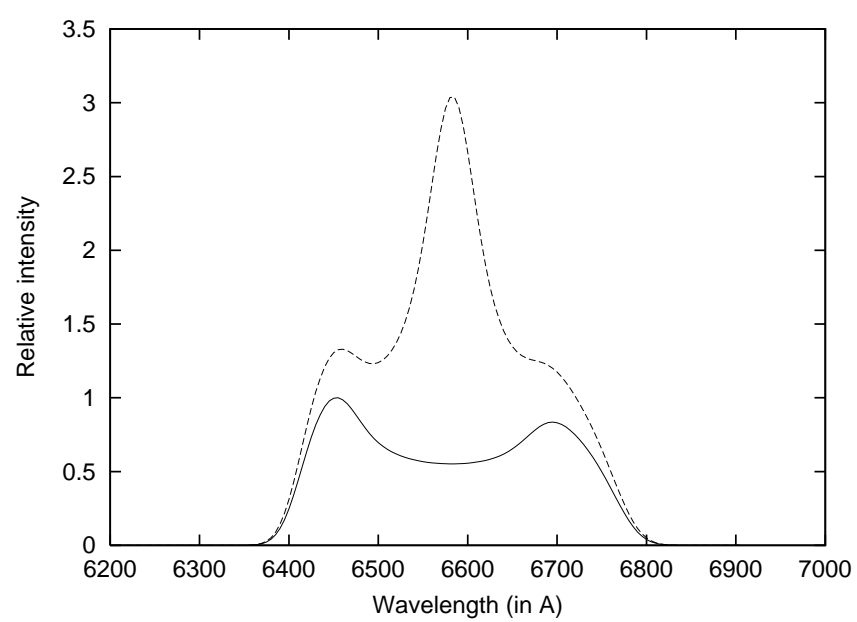

Fig. 6. The same as in Fig. 4 , but for $\phi_{0}=0^{\circ}$.

can be described using a circular or an elliptical photoionized accretion disc model. According to these models, the blue peak of the line should be brighter than the red one (relativistic boosting). Such a trend has been found in

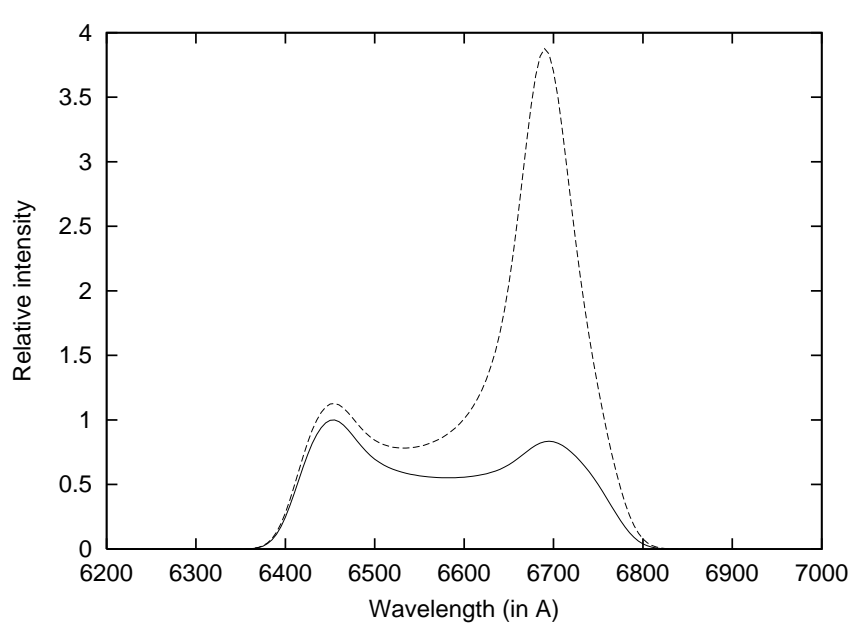

Fig. 7. The same as in Fig. 4 , but for $\phi_{0}=45^{\circ}$.

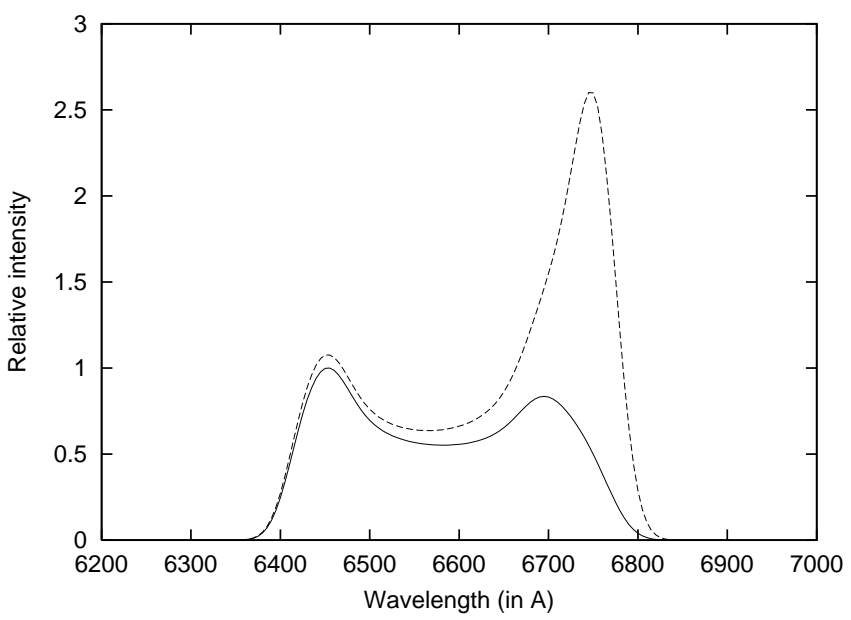

Fig. 8. Same as in Fig. 4, but for $\phi_{0}=90^{\circ}$.

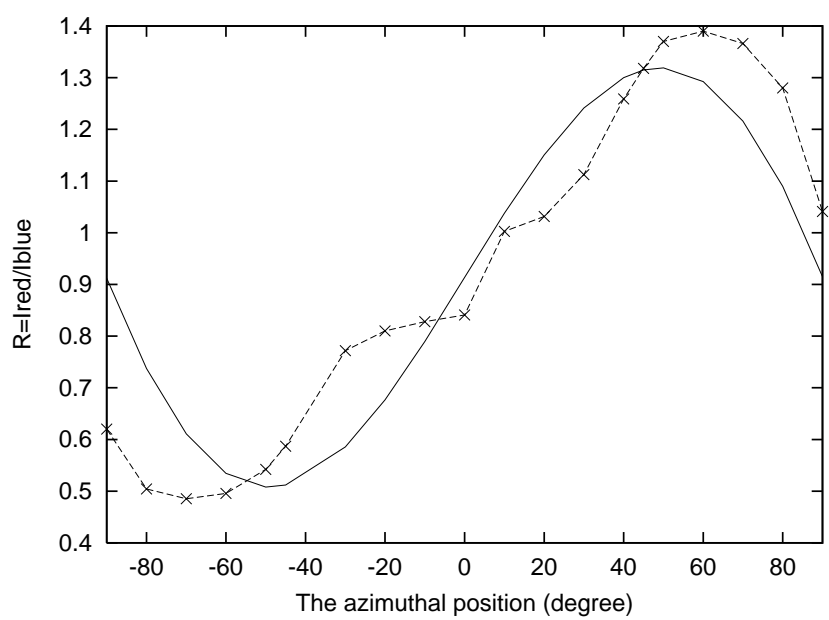

Fig. 9. The ratio of red to blue intensities $(R)$ as a function of azimuthal position of the microlens $\left(\phi_{0}\right)$. The calculations (stars) were performed for a relativistic disc with parameters $i=32^{\circ}, \xi_{\text {in }}=350 \Re_{\mathrm{g}} \xi_{\text {out }}=1000 \Re_{\mathrm{g}}, \sigma / \lambda_{0}=850 \mathrm{~km} \mathrm{~s}^{-1}$. The parameters of the gravitational microlens are $\xi_{0}=500 \Re_{\mathrm{g}}$ and $\eta_{0}=150 \Re_{\mathrm{g}}$. The solid line represents the best fit by a periodical function (Eq. (6)). 


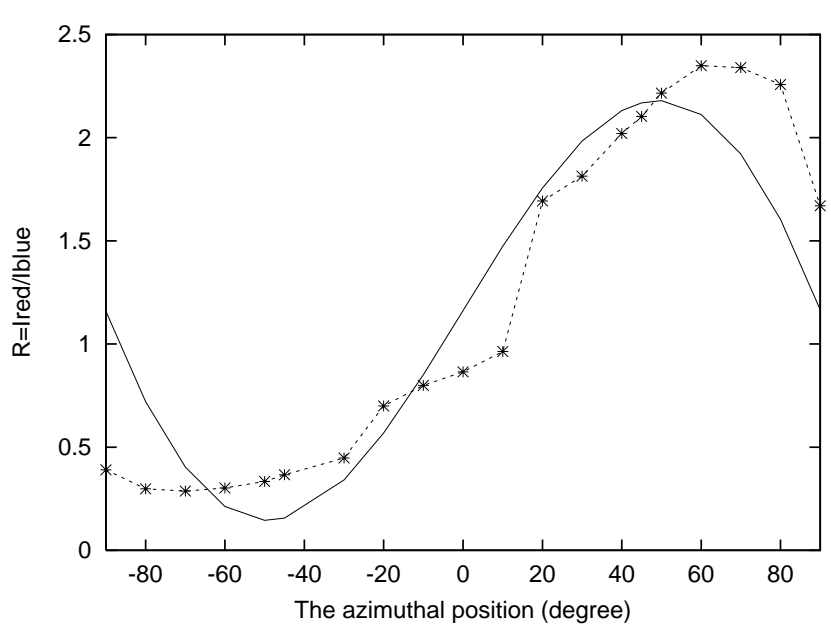

Fig. 10. The same as in Fig. 9, but for $\eta_{0}=300 \Re_{\mathrm{g}}$.

the spectra of this galaxy by Chen \& Halpern (1989) and Newman et al. (1997). However, Miller \& Peterson (1990) found that, during their observations, the red peak was higher than the blue one. Although Newman et al. (1997) could not confirm this result, they noticed a sinusoidallike variation in the ratio of red- to blue-peak intensities in the period from 1990 to 1995 . This variation was confirmed by Sergeev et al. (2000). Similar quasi-periodical variations were also observed in the spectra of 3C 390.3 (e.g. Veilleux \& Zheng 1991; Dietrich et al. 1998). Newman et al. (1997) explained the variability in terms of an accretion disc model with a hot spot, while Sergeev et al. (2000) prefer a model in which the BLR is formed by thousands of clouds rotating predominantly in, but not confined to, the same plane.

As we have shown in the previous section, microlensing can also give rise to relative enhancements between the red and blue peaks of the emission line profile. In fact, we have shown that the transit of a microlens along the accretion disc may induce sinusoidal-like variations of the ratio of red- to blue-peak intensities. In this section, we are going to explore two different physical scenarios in which microlensing could reproduce the behavior of the observed variability in Arp 102 B.

\subsection{Microlensing by a supermassive companion}

In the first place we will address the case of an AGN formed by a supermassive binary. For simplicity, we can consider there to be only one BLR, an accretion disc, located around the primary supermassive black hole. The (less massive) companion will play the role of a microlens during its orbital transit along the accretion disc.

We found that for $\xi_{0}^{1}=\xi_{\text {in }}=350 \Re_{\mathrm{g}}$ a microlens with $\eta_{0} \approx 55 \Re_{\mathrm{g}}$ can produce the observed variation of around $16 \%$ in the red to blue peak ratio in Arp 102 B. However, for $\xi_{0}^{1}=\xi_{\text {out }}=1000 \Re_{\mathrm{g}}$ an ERR of about $160 \Re_{\mathrm{g}}$ is needed. Thus, in the following computations we take $100 \Re_{\mathrm{g}}$ as reference value for the ERR of the microlens.

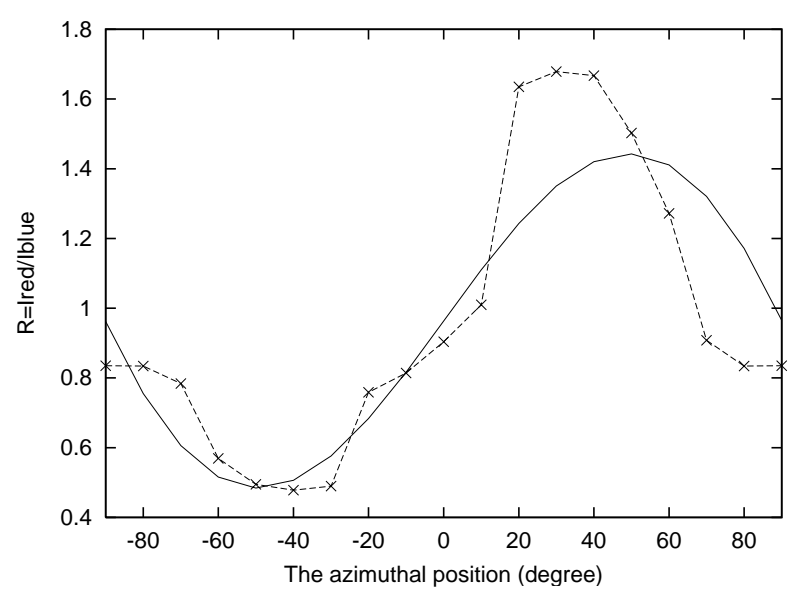

Fig. 11. The same as in Fig. 9, but for $\eta_{0}=200 \Re_{\mathrm{g}}$. The different values of $\xi_{0}$ are due to motion of the lensing object (see text).

Using a mass of $2.2 \times 10^{8} M_{\odot}$ for the supermassive primary in Arp 102 B (Newman et al. 1997) we obtain the following estimates for the inner and outer radii of the accretion disc:

$\xi_{\text {in }}=350 \Re_{\mathrm{g}}=0.00369 \mathrm{pc}$,

$\xi_{\text {out }}=1000 \Re_{\mathrm{g}}=0.0105 \mathrm{pc}$,

for its dimension,

$\xi_{\text {out }}-\xi_{\text {in }}=650 \Re_{\mathrm{g}}=0.00630 \mathrm{pc}$,

and for the Einstein ring radius associated with the microlens,

$\eta_{0}=100 \Re_{\mathrm{g}}=0.00105 \mathrm{pc}$.

From this value of $\eta_{0}$ we can estimate the mass of the microlens. Since the distance, $D$, between the microlens and the accretion disc is negligible with respect to the distance between the observer and the microlens, the expression for $\eta_{0}$ can be approximated as

$\eta_{0} \approx \sqrt{\frac{4 G M_{\mathrm{s}}}{c^{2}} \cdot D}$

From Eq. (7) the mass of the microlens can be estimated as

$M_{\mathrm{s}} \approx \frac{\eta_{0}^{2}}{D} \cdot \frac{c^{2}}{4 G}=5.23 \times 10^{12} \cdot \frac{\eta_{0}^{2}}{D} M_{\odot}$,

where $\eta_{0}$ and $D$, are given in parsecs. In the case of Arp $102 \mathrm{~B}$ we obtained above that $\eta_{0} \approx 0.00105$ pc. Selecting distances between the accretion disc and the microlens in the interval 0.3 to $1 \mathrm{pc}$, we obtain masses for the microlens in the following range:

$M_{\mathrm{s}} \approx 5.8 \times 10^{6} M_{\odot}-2 \times 10^{7} M_{\odot}$.

These masses are an order of magnitude or less than the primary mass. Considering a circular orbit for the companion, the time of transit along the accretion disc is around 
a few years. This order of magnitude is in good agreement with the period of the observed variations, and we can consider this scenario as a plausible explanation of the sinusoidal-like variation in the ratio of red- to blue-peak line fluxes. However the time needed for the companion to complete a circular orbit is around one thousand years; for this reason the observed variations would disappear and then reappear after this long period of time. This represents a strong constrain on this model and can be easily addressed with new observations of the system. On the other hand, with a mass an order of magnitude smaller than the primary, the contribution of the secondary to the emission is too small to change the line profile (Popović et al. 2000).

\subsection{Microlensing by a star-sized object in an intervening galaxy}

An alternative scenario to reproduce the observed variation is the microlensing produced by a star-sized object in an intervening galaxy. The Einstein radius needed $\left(\sim 10^{-3} \mathrm{pc}\right)$ can be produced by an object with $\sim 1 M_{\odot}$ located at a distance around twenty times smaller than the that of the source. Such an object with a relative velocity of around two hundred $\mathrm{km} \mathrm{s}^{-1}$ could also reproduce the observed variations, but in this case they would be observed randomly with a frequency depending on the density of micro-deflectors in the intervening galaxy.

Leaving aside the observed variability of the BEL profiles of Arp $102 \mathrm{~B}$, this result is very important from a general point of view, for we have found that a detectable distortion of the BELs of Arp 102 B (which are well reproduced by an accretion disc model) could be induced by a microdeflector of mass within the typical range of observed microlens masses (e.g. Alcock et al. 2000). Thus, in so far as the BELs of QSOs originate in an accretion disc, the BELs of multiply imaged QSOs should be considered as potentially susceptible to distortion by microlensing effects.

\section{Conclusions}

In this paper we have developed a relativistic accretion disc model, based on that of Halpern \& Chen (1989), for generating line profiles incorporating microlensing. Using this model we have studied the influence of microlensing in spectral line shapes. The following conclusions are worthy of mention:

1. Gravitational microlensing can induce significant changes in the spectral line profiles generated by an accretion disc, even for relatively small values of the Einstein ring radius associated with the microlens $\left(\eta_{0} \simeq 40 \Re_{\mathrm{g}}\right)$. Off-centered microlenses can induce relative enhancements of the blue and red peaks of the line profile, or even give rise to a central peak. These effects could be very strong when $\eta_{0}$ is comparable to the disc dimension, $\xi_{\text {out }}-\xi_{\text {in }}$.
2 . We found that a compact object of $\sim 1 M_{\odot}$ in an intervening galaxy could give rise to effects easily detectable in the emission line profiles. That implies that, contrary to what has been assumed, not only the continuum but also the emission lines may be susceptible to microlensing in multiply imaged QSOs (in so far as the accretion disc model for Arp 102 B applies to a typical BLR).

3 . The transit of a microlens along the accretion disc can qualitatively reproduce the quasi-periodical variability of the ratio of red-to-blue peak intensities observed in the case of Arp 102 B in the 1990-1995 period.

4. We have explored two different physical scenarios that could reproduce the observed variability in Arp $102 \mathrm{~B}$ : i) microlensing by a supermassive companion $(M \approx$ $10^{7} M_{\odot}$ ) located at a distance of around $0.3 \mathrm{pc}$, and ii) microlensing by a compact object of mass $\sim 1 M_{\odot}$ in an intervening galaxy. The sinusoidal-like variation induced by microlensing does not extend beyond one period. It can be reproduced again after a long lapse of time (in the case of the supermassive companion) or randomly (in the case of the microdeflector in an intervening galaxy). This could be useful in distinguishing between microlensing and other different options that are strictly periodical, such as the model of an accretion disc with a hot spot.

Acknowledgements. This work is a part of the project P6/88 "Relativistic and Theoretical Astrophysics" supported by the IAC and "Astrophysical Spectroscopy of Extragalactic Objects" supported by the Ministry of Science and Technology of Serbia. L Č. Popović thanks the IAC for its hospitality during his visit.

\section{References}

Abajas, C., Mediavilla, E. G., Muñoz, J. A., Popović, L. Č., \& Oscoz, A. 2001, in preparation

Agol, E., \& Krolik, J. 1999, ApJ, 524, 49

Alcock, C., et al. 2000, ApJ, 542, 281

Antonucci, R., Hurt T., \& Agoi, E. 1996, ApJ, 456, L23

Belle, K. E., \& Lewis, G. F. 2000, PASP, 112, 320

Chen, K., \& Halpern, J. P. 1989, ApJ, 344, 115

Chen, K., Halpern, J. P., \& Filippenko, A. V. 1989, ApJ, 339, 742

Corbett, E. A., Robinson, A., Axon, D. J., Young, S., \& Hough, J. H. 1998, MNRAS, 296, 721

Dietrich, M. Peterson, B. M., Albrecht, P., et al. 1998, ApJ, 115,185

Eracleous, M., \& Halpern, J. 1994, ApJS, 90, 1

Eracleous, M. 1999, in Structure and Kinematics of Quasar Broad Line Regions, ed. C. M. Gaskell, W. N. Brandt, M. Dietrich, D. Dultzin-Hacyan, \& M. Eracleous, ASP Conf. Ser., 175, 163

Eracleos, M., Halpern, J. P., Gilbert, A. M., Newman, J. A., \& Filippenko, A. V. 1997, ApJ, 490, 216

Gaskell, C. M. 1996, ApJ, 464, L107

Halpern, J. P., \& Filippenko, A. V. 1988, Nature, 331, 46

Halpern, J. P., Eracleous, M., Filippenko, A. V., \& Chen, K. 1996, ApJ, 464, 70

Irwin, M. J., Hewett, P. C., Corrigan, R. T., Jedrzejewski, R. I., \& Webster, R. L. 1989, AJ, 98, 1989

Kayser, R., Refsdal, S., \& Stabell, R. 1986, A\&A, 166, 36 
Kraus, A., Quirrenbach, A., Lobanov, A. P., et al. 1999, A\&A, 344,807

Miller, J. S., \& Peterson, B. M. 1990, ApJ, 361, 98

Mineshige, S., \& Yonehara, A. 1999, PASJ, 51, 497

Nemiroff, R. J. 1988, ApJ, 335, 593

Newman, A. J., Eracleous, M., Filippenko, A., Halpern, J. P. 1997, ApJ, 485, 570

Ostriker, J. P., \& Vietri, M. 1985, Nature, 318, 446

Perez, E., Mediavilla, E., Penston, M. V., Tadhunter, C., \& Moles, M. 1988, MNRAS, 230, 353

Popović, L.Č., Mediavilla, E. G., \& Pavlović, R. 2000, Serb. Astron. J., 162, 1

Popović, L. Č., Stanić, N., Kubičela, A., \& Bon, E. 2001, A\&A, 367,780
Schneider, P., \& Weiss, A. 1987, A\&A, 171, 49

Schneider, P., \& Wambsganss, J. 1990, A\&A, 237, 42

Sergeev, S. G., Pronik, V. I., \& Sergeeva, E. A. 2000, A\&A, 356,41

Sulentic, J. W. 1999, Structure and Kinematics of Quasar Broad Line Regions ed. Gaskell, C. M., Brandt, W. N., Eracleous, M., Dietrich, M., \& Dultzin-Hacyan, D., ASP Conf. Ser., 175, 175

Veilleux, S., \& Zheng, W. 1991, ApJ, 377, 89

Wandel, A., Peterson, B. M., \& Malkan, M. A. 1999, ApJ, 526, 579

Yonehara, A., Mineshige, S., Fukue, J., Umemura, M., \& Turner, E. L. 1999, A\&A, 343, 41

Zheng, W., Veilleux, S., \& Grandi, S. A. 1991, ApJ, 381, 418 